\title{
La Enseñanza del Algebra y la Reprobación. Una Experiencia para Compartir.
}

\author{
Hernández Méndez, Griselda \\ Universidad Veracruzana, México \\ griseldahm2001@yahoo.com.mx \\ Mendoza López, Alejandro \\ Universidad Veracruzana, México \\ amendoza@uv.mx
}

\author{
Hernández Méndez, Edith \\ Universidad de Quintana Roo, México \\ hernandez147@gmail.com
}

Resumen - El presente artículo es resultado de una investigación aplicada en dos escuelas secundarias; cuyo propósito fue disminuir los índices de reprobación en Matemáticas, a través del diseño e implementación de una propuesta didáctica basada en el uso de las TIC y sustentada en el constructivismo. En este texto se narra una de las experiencias vividas en un aula, en la escuela Secundaria Julio Zárate. Se muestra cómo fueron las reacciones de los estudiantes hacia esquemas diferentes de enseñar y aprender, y cuáles fueron los resultados obtenidos concernientes al aprendizaje del álgebra.

Palabras claves - Aprendizaje; Constructivismo; Propuesta didáctica; Estrategias;

Abstract - This paper is the result of an applied research conducted at two middle schools in Xalapa (Mexico) whose purpose was to decrease the failure rate of a second grade math course, by means of a didactic proposal based on new information and communication technologies(ICT), and an eclectic constructivist approach; sensible to the set objectives. Given the scope of this research, in this paper, we narrate one of the experiences we went through in a classroom where the proposal was carried out at the middle school Julio Zárate. Our aim is to report the findings regarding the learning of algebra.

Keywords - Learning; Constructivism; Didactic proposal; strategies;

\section{INTRODUCCIÓN}

Este texto se constituye en informe narrativo de los resultados de una indagación que se realizó, a partir de la aplicación de una propuesta didáctica basada en el uso de las TIC y sustentada en el enfoque constructivista, en la escuela secundaria Julio Zárate de la ciudad de Xalapa perteneciente al Estado 
de Veracruz. Los objetivos de dicha investigación fueron: disminuir los índices de reprobación en Matemáticas y, demostrar que las matemáticas, lejos de ser difíciles, pueden aprenderse de manera divertida y significativa. La investigación se efectuó en el año dos mil doce, y partió del siguiente problema, que aquí se esboza:

En el 2006, en las pruebas PISA (Programa para la Evaluación Internacional de Alumnos de la OCDE) de matemáticas, México obtuvo 392 puntos de 800 posibles. Se pretendía que en 2012 el puntaje fuera de al menos 435; sin embargo, esto no se logró.Aunque la Reforma de la Educación Secundaria (RES), impulsada por la pasada administración, se comprometió a abatir los rezagos en ese nivel, aún continúan altos índices de reprobación en asignaturas como matemáticas $\mathrm{e}$ inglés, reveló el Informe Nacional de Seguimiento a las Escuelas, ciclo escolar 2006-2007, de la Secretaría de Educación Pública (SEP).

Indiscutiblemente, la matemática es una herramienta potente y eficaz en la interpretación y, en su caso, en la resolución de multitud de pro- blemas, fenómenos y situaciones de todo tipo. La misma historia nos muestra el enorme apoyo que las matemáticas han brindado al desarrollo de grandes inventos, entre éstos destacan: la computadora, las leyes de la física, fórmulas químicas... De tal manera, que las matemáticas se asocian al desarrollo económico, tecnológico e incluso político y cultural de los países. Está demás decir que el adecuado aprendizaje de las matemáticas es indispensable en un país como el nuestro que establece lazos con países desarrollados. No obstante, los marcados índices de reprobación son desalentadores.

A pesar de que se han realizado diversas investigaciones en torno a las causas que provocan la reprobación y/o el bajo rendimiento en la materia de matemáticas, éstas no han impactado suficientemente para disminuir la reprobación y elevar el rendimiento. Son múltiples los factores que intervienen en esta problemática, intrínsecos (psicológicos) y extrínsecos al estudiante (pobreza, rezago, desintegración familiar, entre otros). Pero quizás el de mayor envergadura sea el problema que implica el 
acercamiento a las matemáticas; son recurrentes las quejas de los estudiantes por no comprenderlas, así como las de los maestros para quienes, desde su percepción, los alumnos son cada vez más apáticos y renuentes al estudio de las matemáticas.

Bajo esas consideraciones, los investigadores que participamos en esta indagación, presuponíamos que la reprobación en matemáticas es multifactorial y multidimensional, pero sostuvimos que una gran cantidad de estudiantes reprueban por no comprender las matemáticas, causa que podría ser generada por el paradigma de enseñanza del profesorado.

La matemática es una disciplina dura, lógica y, aunque es aplicada, generalmente en las aulas se enseña de manera abstracta, sin aterrizarla a lo concreto real y sin recurrir a los conocimientos previos de los alumnos. Por eso muchos estudiantes manifiestan cierta fobia hacia esta materia.

En el periódico la Gaceta $\left(1^{\circ} \mathrm{de}\right.$ julio de 2002) aparece un texto que declara que la fobia hacia las ma- temáticas se presenta desde la infancia, pues en México no existe una cultura de las matemáticas, ya que se cree erróneamente que esa disciplina es sólo para genios. Así, tanto la sociedad como el profesorado se han encargado de divulgar esa falacia: "las matemáticas son difíciles".

Las matemáticas, lejos de ser difíciles, pueden aprenderse de manera divertida y significativa. La desfavorable imagen heredada hacia las matemáticas se ve fortalecida por los procedimientos didácticos usados por la mayoría de los docentes, y por sus actitudes poco flexibles, que sólo consiguen obstruir la alegría de descubrir y construir.

De ese modo, nos atrevimos a elaborar una propuesta original de enseñanza de las matemáticas y llevarla a las aulas, bajo los sustentos de un constructivismo ecléctico, pero circunspecto, que se apoyara en estrategias constructivistas y en los recursos de las nuevas tecnologías de la información y de la comunicación. 


\section{SUSTENTOS TEÓRICOS}

Teníamos claro que constructivismo es análogo a una sopa conceptual al hacer referencia a la fundamentación epistemológica, a las distintas teorías psicológicas que tienen en común estos planteamientos, a los desarrollos educativos en el aula, a las estrategias didácticas $y$ hasta a los profesores y profesoras que los utilizan (Rodrigo y Cubero, 1998). Ponderamos por una enseñanza creativa, recreativa, viva, emotiva; amparándonos en diversos intelectuales de la educación, como los pedagogos de la escuela nueva: Decroly, Freinet, Montessori, Dewey, entre otros, quienes defienden la idea de una enseñanza centrada en el alumno (paidocentrismo) y conciben a dicho alumno como un ente pensante activo, inteligente, con intereses específicos y necesidades; jamás pasivo ni reactivo.

Para estos autores, el paradigma de enseñanza tradicional ha provocado lo que Freinet (1964) llamó anorexia educativa, pues el exceso de contenidos al que se ven sometidos los alumnos conlleva a que no aprendan por gusto, ni le encuen- tren sentido al aprendizaje, de tal manera que expelen los contenidos como especies de autómatas.

Dewey (1958), por su parte, señaló la importancia sustancial del reconocimiento de los intereses y experiencias de los alumnos, lo cual es tan importante como los conocimientos previos tan reiterados por los constructivistas. Si éstos se enganchan con el nuevo material para revisarse, seguramente adquirirán sentido para el alumno. Para nosotros tuvo especial interés la teoría cognitiva y la humanista centrada en el aprendizaje. Ambos enfoques teóricos plantean a los maestros nuevas formas de enseñar y de relacionarse con los estudiantes:

Primero, que el aprendizaje no es la simple asimilación de conocimientos. "Aprender es una consecuencia de pensar... "la retención, la comprensión y el uso activo del conocimiento sólo pueden lograrse mediante experiencias de aprendizaje en las que los alumnos piensan acerca de y con lo que están aprendiendo" (Perking, 1995, p.55). 
Segundo, que se requieren ciertas cualidades para enseñar matemáticas, como:

a) Entusiasmo. Definido por el interés mostrado hacia la materia que se enseña y se denota incluso con las miradas, gestos, voz, énfasis (Hernández, 2012);

b) Empatía. Desde el punto de vista rogersiano (Roger, 1991), la empatía significa colocarse en el lugar del alumno para comprender su punto de vista. Pareciera que el maestro se olvidó que una vez fue alumno y que a veces no entendía algunos temas y que también se aburría en las clases de maestros expositivos;

c) Expectativas positivas. Para el enfoque rogersiano (Roger, 1991), es trascendental confiar en las posibilidades del alumno; es decir, ayudarles a desarrollar un "yo positivo. Las expectativas son inferencias que los maestros hacen acerca de los logros académicos de los alumnos, basados en lo que sabe ahora de ellos (Good y Brophy 1994, en Hernández et al, 2012). A veces, los maestros son discriminativos, ya que marcan diferencias entre los estudiantes, distinguiendo a "los buenos en matemáticas de los malos".

d) Calidez humana. Es importan- te que el profesor comprenda que trabaja con personas con sentimientos y emociones $y$. que, ante la crisis de valores, vivimos en un mundo carente y necesitado de amor. Los alumnos requieren ser tratados como personas y no como simples aprendices. El rapport, ambiente humano, es urgente, más aún que las decoraciones artificiales de los salones.

Permanecer tantas horas en las aulas y en un ambiente hostil, aflige a cualquiera. ¿Quién quisiera entrar a la clase de matemáticas de un maestro que genera miedo?

Tercero. Para nuestra propuesta fueron esenciales las habilidades docentes para lograr aprendizajes significativos, como:

a) Conocimiento y manejo de la disciplina; empleo de una metodología de enseñanza constructivista, creativa y adaptada a los intereses y necesidades del estudiantado (Hernández, Juárez, Landgrave y Mendoza, 2012).

b) Facilitación de los aprendizajes. Implica abandonar la idea de que los alumnos son neófitos y el maestro erudito. Es conveniente renunciar al papel de guías, participando sólo como acompañantes en el proceso de aprendizaje. 
c) Ser andamios, puentes entre los conocimientos que el alumno trae al aula (conocimientos previos) con los nuevos a adquirirse.

d) Ser creativos para enfrentar los imprevistos y para despertar el interés, la imaginación y la construcción de conocimientos y saberes.

e) Tener habilidad para utilizar las nuevas Tecnologías de la Información y la Comunicación (TIC).

f) Hacer encabalgamiento: Se refiere a la capacidad de atender dos asuntos o más, prestar atención a las distracciones sin entorpecer las actividades de aprendizaje. Supone dos actividades de instrucción; verbigracia, supervisar los ejercicios de matemáticas y atender a un estudiante que se acerca a preguntarle cuando tiene dudas.

g) Retroalimentar y evaluar formativamente. Es imperativo que el profesor esté al pendiente de los logros y avances de sus alumnos, retroalimentando y evaluando constantemente de manera formativa y no únicamente informativa.

Del mismo modo, las estrategias de enseñanza constructivista fueron esenciales en la conformación de nuestra propuesta. Habilidades-es- trategias constituyeron un binomio esencial para alcanzar los propósitos que perseguíamos.

Las habilidades son el conjunto de destrezas del que dispone el maestro; son capacidades que pueden expresarse en conductas en cualquier momento, porque han sido desarrolladas a través de la práctica y que, además, pueden utilizarse o ponerse en juego, tanto consciente como inconscientemente, de forma automática. En cambio, las estrategias se utilizan de forma consciente. La estrategia se considera como una guía de las acciones que hay que seguir, y obviamente, es anterior a la elección de cualquier otro procedimiento para actuar.

La estrategia en sí misma, por buena que sea, no va a surtir efecto si el maestro carece de la habilidad necesaria para su desarrollo en clase. Se requiere, como en todo, conocer por qué, cuándo, para qué y cómo emplear esta estrategia. En otras palabras, desarrollar la competencia profesional que su aplicación exige. 
En ese sentido, como parte de las estrategias de enseñanza, en el aula se implementaron las tecnologías que estuvieran ad hoc con los contenidos, objetivos y actividades de aprendizaje seleccionados.

En la investigación general, por supuesto, se profundizó en el constructivismo, conceptos de aprendizaje, esquemas, pero por efectos de espacio, no podemos explicar todo.

\section{MÉTODO}

A partir de la hipótesis de que la implementación de la propuesta de enseñanza constructivista con énfasis en el uso de las TIC, disminuiría el índice de reprobación en matemáticas de los estudiantes de segundo grado de las escuelas secundarias, al mismo tiempo que ellos aprenderían de manera divertida las matemáticas; la investigación se desarrolló en tres fases: en la primera, una vez elaborado el marco teórico y referencial, se diseñó la propuesta didáctica congruente con todo lo planeado y argumentado. La segunda fase consistió en la formación de los investigadores (cuatro investigadores quienes con- taban con experiencia y conocimientos matemáticos) que aplicarían la propuesta en las aulas de secundaria seleccionadas. Finalmente, en la tercera fase, se aplicó la propuesta, se llevó seguimiento de los procesos y se validó su alcance a partir de los resultados de aprendizaje y de las respuestas de los alumnos.

La fase dos, que básicamente se constituyó en un seminario formativo, fue esencial porque no todos los que aplicarían la propuesta contaban con formación pedagógica. Aquí nos percatamos lo "fácil que es repetir lo que dicen los autores del enfoque constructivista, pero también lo difícil que es aplicarlo en el aula" (Hernández et al, 2012). Por eso se invitaron a expertos en el tema y a estudiantes de segundo grado de secundaria a participar en el seminario y a ellos, expertos y estudiantes, les mostrábamos cómo enseñábamos el tema con las estrategias elegidas. Nos cerciorábamos si los alumnos comprendían y se enrolaban con el tema. Por fortuna, fueron excelentes retroalimentadores y fuertes críticos cuando no estaban de acuerdo con las formas de enseñar. 
Para la tercera fase -implementación de la propuesta y la medición de su valía- se utilizó el método básico de los estudios cuasi experimentales, apto para estudiar problemas en los cuales no se puede tener absoluto control, en este caso, la reprobación en matemáticas, pero se pretende una vez intervenido, tener el mayor control posible. Otra de sus características es que se trabaja con grupo "intactos"; es decir, ya establecidos, y es recomendado aplicar una preprueba, una medición previa a la intervención, a fin de analizar su paridad con otros grupos (los grupos controles o experimentales fueron a los que se les implementó la propuesta y los grupos testigos fueron de apoyo para aplicarles el examen final y comparar sus resultados con los primeros).

\section{Muestra seleccionada.}

Para la investigación amplia, se aplicó la propuesta en dos secundarias de Xalapa: Julio Zárate y David Alfaro Siqueiros, turno matutino y vespertino. Solo cuatro grupos fueron seleccionados, vía procedimiento intencional, puesto que los cuatro directores de ambas escuelas, en sus dos turnos, fueron quienes con agrado aceptaron participar en el cuasiexperimento y ellos nos asignaron a los grupos.

\section{Procedimiento: diseño e im- plementación de la propuesta.}

Coincidentemente, los directores de las escuelas decidieron que el cuasiexperimento se llevara a cabo en la fecha próxima que correspondería al tema de Álgebra, porque, además, era el que mostraba mayores índices de reprobación. El contenido originalmente se planteó para impartirse en dos meses, sin embargo, los directores nos permitieron trabajar en las aulas solamente quince días, para evitar conflictos con supervisores o sindicatos.

Fue así como procedimos a la construcción de la propuesta didáctica de álgebra, atendiendo tres lapsos, con base en el momento en que se utilizan las estrategias constructivistas: antes de la instrucción, durante y después de la instrucción (Cfr. Díaz Barriga y Hernández, 2002). 
Casi al mismo tiempo que se elaboraba la propuesta, diseñamos, junto con un experto en tecnología, un software práctico y entretenido para reforzar saberes básicos de álgebra. Adicionalmente, se preparó un $C D$ con ejercicios que los estudiantes llevarían a casa para practicar. La tecnología fue un recurso importante, como el uso de fotos en Internet, de cantantes famosos, para resolver problemas que implicaran adición y sustracción de expresiones algebraicas. El chat para intercambiar información sobre novios y amigos, edades... todo con el fin de enlazar esto con expresiones algebraicas.

Emplear la música, acompañarnos de grabadoras y micrófonos para cantar los números enteros, adiciones, sustracciones, multiplicaciones y divisiones, fueron otras de las formas implementadas en el aula.

\section{"Aprendiendo matemáticas con} Karen", es otro programa en CD, parecido a "Aprendiendo con PIPO", pero el primero lo elaboró nuestro equipo, cuya finalidad fue acompañar divertidamente a los adoles- centes en el conocimiento de las matemáticas. Aprenden, entre otras cosas, a estimar y medir ángulos, a representar con literales valores desconocidos, a resolver problemas que impliquen el planteamiento y la resolución de ecuaciones, etc. Cabe decir que la investigación contó con recurso del Consejo Veracruzano de Ciencia y Tecnología (COVECYT).

Para este artículo, se muestran algunos de los resultados, que más bien se constituyen en reflexiones narradas de lo experimentado en el aula de segundo grado, grupo $D$, del turno vespertino, de la secundaria Julio Zárate. ¿Por qué la narración? Porque generalmente muchos sucesos en las escuelas no son comentados; es como si careciera de sentido elucidar inclusive lo cotidiano. Sostenemos que contar lo vivido, crearlo y recrearlo... es análogo a revivir lo que se cree simple, pues solamente haciéndolo suyo se puede dar cuenta de las dificultades, de los tropiezos, de los errores y de los logros y deseos, esfuerzos... (Hernández et al, 2012). 


\section{RESULTADOS}

Una experiencia para comentar...

Por dificultades de espacio no podremos comentar todo respecto a lo que se hizo y experimentó en el aula, así que nos concentraremos en algunas de las actividades.

Debíamos trabajar el tema: significado y uso de las literales y como subtema: ecuaciones. Los conocimientos y habilidades a desarrollar eran: resolver problemas que impliquen el planteamiento y la resolución de ecuaciones de primer grado de la forma $\mathrm{ax}+\mathrm{bx}+\mathrm{c}=\mathrm{dx}+\mathrm{ex}+$ $\mathrm{f}$, con paréntesis en uno o en ambos miembros de la ecuación, utilizando coeficientes enteros o fraccionarios, positivos o negativos.

De esa manera, las intenciones didácticas fueron que los alumnos: Reflexionaran sobre la similitud entre una balanza en equilibrio y una igualdad en la que se desconoce un valor, encontrarán el valor de una incógnita de una ecuación y resolvieran problemas, a través del planteamiento y resolución de ecuaciones de primer grado, con una incógnita.
En tanto que nuestra intención también era hacer amenas y divertidas las clases, buscamos estrategias didácticas adecuadas a la edad de los estudiantes, empleamos técnicas grupales y utilizamos materiales diferentes como matamoscas, globos, pancartas, entre otros. Ya preparado todo el material para las actividades, nos presentamos en las instalaciones de la escuela para dar inicio a las clases. Encontramos un ambiente cordial, gran disposición y apertura por parte del maestro responsable del curso, el cual nos brindó todas las facilidades.

El primer día, después de la presentación por parte del titular del curso, procedimos a comunicar y consensuar las reglas básicas para el buen funcionamiento del curso y poder lograr así los mejores resultados. Platicamos con ellos sobre la importancia del conocimiento de todas las materias en general $y$, en especial, de las matemáticas para la vida cotidiana de todos los seres humanos. Recuperamos sus saberes y experiencias previas. 
El salón de clases, como muchos otros que existen en las escuelas de la ciudad de Xalapa, resulta pequeño para albergar a más de cuarenta alumnos del grupo. El mobiliario está formado por sillas y mesas individuales, tan pesadas que se dificulta su movimiento o traslado de un lugar a otro. El maestro tiene un escritorio y silla sobre un templete de cemento; también cuenta con un pizarrón de acrílico y no tiene tomacorriente o contactos para enchufar aparatos electrónicos. A pesar de estas circunstancias desfavorables (que ciertamente entorpecieron nuestra tarea), nos dispusimos a iniciar las actividades con gran entusiasmo.

El examen diagnóstico. Punto de inicio para los cuasi experimentos.

Se procedió a aplicar un examen diagnóstico para determinar el grado de conocimiento de los alumnos. De esa manera, el examen diagnóstico, elaborado por el equipo, constó de quince reactivos: siete numéricos, tres geométricos, tres de álgebra, un reactivo de concepto algebraico y uno de concepto geométrico.
Se encontró, con base en los resultados del examen diagnóstico, que los conocimientos previos eran deficientes en la mayoría de los casos, puesto que se obtuvieron 41 calificaciones bajas, 38 reprobatorias y tres aprobatorias. Solamente dos alumnos obtuvieron calificaciones altas. Esto significa que, de un total de quince aciertos posibles, dos de los alumnos lograron doce aciertos en el caso de la calificación más alta con un promedio de 8.0 , y uno de los alumnos obtuvo dos aciertos en el caso de la calificación más baja con un promedio de 1.33 .

De estos ejercicios contestados correctamente por más del $50 \%$ de los alumnos, uno es aritmético, tres son geométricos y uno es algebraico. Se esperaba que todos, o al menos la mayoría de los alumnos, contestaran correctamente los reactivos de tipo aritmético, pues son de nivel básico para posteriores saberes.

\section{Desarrollo de las clases.}

Al siguiente día nos presentamos con una balanza hecha con diversos materiales tales como un porta 
tazas, una barra de madera, dos tapas de plástico y unos pedazos de hilo, así como frijoles, habas, garbanzos y otros objetos. La idea inicial fue encargar a los alumnos la construcción de una balanza hecha con material desechable que encontraran en casa, pero al considerar que se trataba del primer día de actividades, finalmente decidimos mostrarles un artefacto construido por nosotros. Causó gran expectación entre los alumnos el ver sacar del maletín la balanza, preguntando y comentando entre ellos para qué sería utilizada.

Procedimos a jugar con la balan$z a$, señalando de inicio que se encontraba equilibrada. Observando cómo se movía al depositar diferentes objetos en cada uno de los platillos de la balanza, preguntamos e invitamos a los alumnos a participar en el juego para lograr diferentes situaciones de equilibrio, agregando y quitando distintas cantidades de objetos. Inicialmente lo hicimos con varios objetos de manera arbitraria, desordenada; posteriormente lo hicimos con pocos objetos y solicitando a los alumnos que tomaran nota de las situaciones que iban observando. Enseguida se reescribieron las notas en lenguaje matemático utilizando la primera letra de cada objeto y los números para representar las cantidades utilizadas en las diferentes situaciones de equilibrio y usando el símbolo de igual para indicar el símil de los platillos con los lados izquierdo y derecho de una proposición de igualdad o de una ecuación.

Una vez que se aclaró el funcionamiento de una balanza y se tuvo presente la necesidad de vincular los conocimientos teóricos de las matemáticas con el entorno de los estudiantes, de su relación con eventos sociales, naturales y de otras áreas del conocimiento, mencionamos que aquí en el parque Juárez existen cuatro estatuas; los estudiantes señalaron que las habían visto y algunos sabían lo que representan: una de ellas, la cual sostiene entre sus manos precisamente una balanza y tiene los ojos vendados para significar el equilibrio y la imparcialidad, simboliza la Justicia, la cual. Junto con los estudiantes se trajeron a colación otros objetos que asemejan una balanza, como el titiribaco o sube y baja. Observamos que los desequilibrios momentáneos nos 
permiten disfrutar del juego; hablamos del equilibrio ecológico, de cómo la tala inmoderada de los árboles provoca desajustes, desequilibrios que han ocasionado el cambio climático, etcétera. Observamos que las ideas de equilibrio y de igualdad no son privativas de las matemáticas y que éstas se encuentran muy ligadas a nuestra actividad cotidiana; solamente debemos prestar atención y darnos cuenta de que las usamos constantemente.

Posteriormente, procedimos a distribuir una actividad propia de la consigna número 1 del plan de clase correspondiente, a través de la cual se reforzaría el uso de las ecuaciones con base en ejemplos, para resolver problemas basados en una balanza.
El texto y las dos ilustraciones siguientes forman parte de dicho material y se encuentran en los documentos oficiales del curso.

I. La siguiente balanza está en equilibrio.

1. ¿Cuáles de las siguientes acciones la mantendrían en equilibrio? ¿Cómo lo representarías con una expresión algebraica?

a) $\mathrm{Si}$ pesa $3 \mathrm{Kg}$. el platillo izquierdo ¿cuánto pesa el platillo derecho?

b) Añadir $4 \mathrm{Kg}$. a cada platillo.

c) Quitar $5 \mathrm{~kg}$ a cada platillo.

d) Pasar un bote del platillo derecho al platillo izquierdo.

e) Quitar dos botes del platillo izquierdo y un bote del derecho.

f) Quitar un bote de cada platillo.

\section{Imagen 1: balanza en equilibrio.}

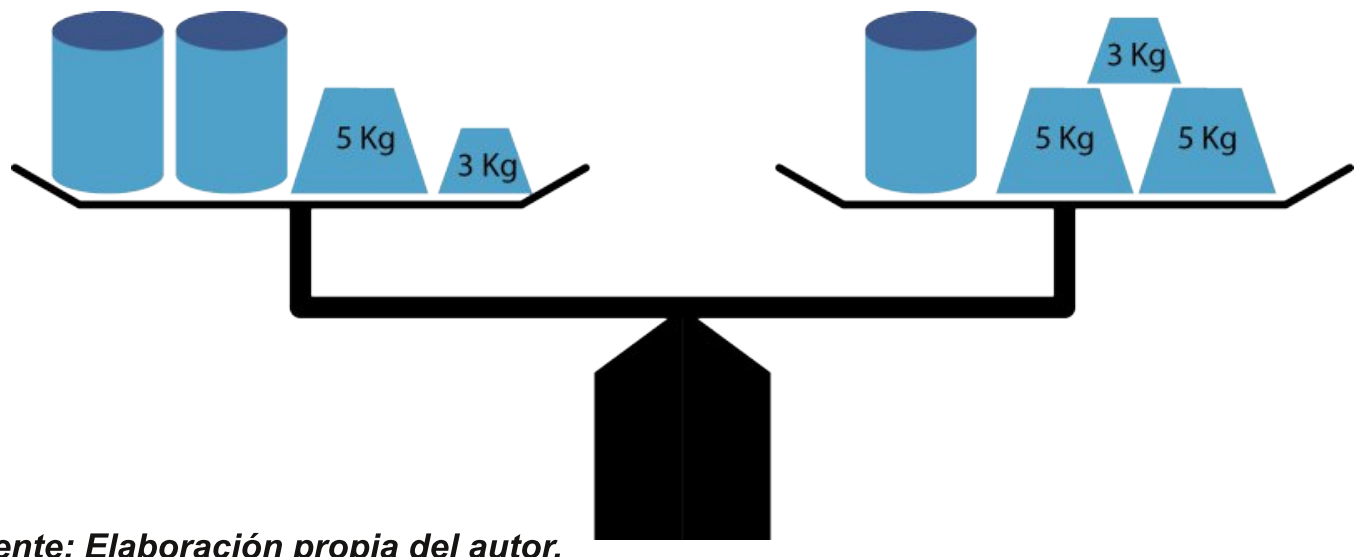

\section{Fuente: Elaboración propia del autor.}


2 botes $+8 \mathrm{~kg}=1$ bote $+13 \mathrm{~kg}$

$2 b+8=1 b+13$

$2 x+8=1 x+13$

Es necesario hacer notar que la agudeza de algunos de los alumnos y el sentido común de la mayoría de ellos les permitieron descubrir que un bote pesa $5 \mathrm{Kg}$, sin necesidad de pedírselo y sin necesidad de escribir ninguna ecuación. Se detectaron varios procedimientos para llegar a la respuesta.

En los siguientes días, se procedió a emplear alguna de las diferentes técnicas didácticas que previamente habíamos planeado en equipo para intentar motivar a los alumnos a participar en la construcción del conocimiento.

Revisión de la consigna número dos. Aquí procedimos, con base en las anotaciones obtenidas de la representación con la balanza, a escribir las ecuaciones correspondientes a cada uno de los dibujos usados para ilustrar la consigna. Para recordar y reforzar los conocimientos teóricos básicos de la aritmética y del álgebra, anota- mos diferentes ecuaciones sugeridas por los alumnos, teniendo cuidado de resaltar las propiedades de la igualdad, de los números y de los signos, según fuese el caso. Esto fue necesario en virtud de los deficientes conocimientos mostrados por los alumnos en el examen diagnóstico. Asimismo, en cada clase se procedió a vincular las matemáticas con situaciones cotidianas. Por ejemplo, se les motivaba a contestar preguntas como "si el litro de leche cuesta 12 pesos y compro 2 litros, ¿cuánto gasté?" Todos contestaron 24 pesos. Después de esto se procedió a escribirlo en lenguaje matemático $2 \mathrm{~L}=24$, lo cual nos llevó a remontarnos a la primaria donde buscábamos el número perdido (12). En la secundaria despejamos $L$ y decimos $L=24 / 2$ : $L=12$, justificando esta respuesta con base en las propiedades de la igualdad.

Otro ejemplo más: "si a Puebla son $270 \mathrm{Km}$. (d) y un autobús tarda 3 horas $(t)$ en llegar ¿Cuál es la velocidad? Pues $90 \mathrm{Km} / \mathrm{h}$, contestaron. ¡Ah! Dijimos entusiasmados "han descubierto una fórmula mágica: $V=d / t$ ", la cual nos permite co- 
nocer la velocidad de un móvil cualesquiera conociendo la distancia (d) y el tiempo (t) empleado en recorrerla.

Lo anterior nos permitió reforzar la idea de que la teoría matemática es accesible para todos y está más cerca de nosotros de lo que habíamos imaginado. Así, mientras nos divertimos buscando otros ejemplos, aprovechamos para ir repasando algunos principios básicos, necesarios para el estudio, la comprensión y manejo de las ecuaciones de primer grado. Ellos fueron muy participativos, querían dar la respuesta lo más rápido posible. Nos percatamos que las concepciones de muchos profesores en torno a que los alumnos son apáticos y no desean aprender, es quizás una falacia, al menos allí estaban entusiasmados, con risas y aplausos constantes.

Siguiendo con los planes de clase, al día siguiente se pidió a los estudiantes una actividad, que se realizaría en ocho equipos de cinco alumnos cada uno. La actividad consistió en resolver unos problemas, como los siguientes:
Andrea tiene una colección de 654 estampillas y por accidente perdió $2 / 5$ de ellas ¿Cuántas estampillas perdió?

De 550 aves que tiene una granja 2/5 son gallinas; el resto son patos ¿Cuántas aves hay de cada especie?

Mi papá es albañil y cobra $\$ 2,500$ pesos por levantar un muro de $15 \mathrm{~m} 2$ ¿Cuánto cobrará por construir un muro de $45 \mathrm{~m} 2$ ¿Cuánto por un muro de $70 \mathrm{~m} 2$ ?

Cada uno de los equipos debería resolver el problema y escribirlo de forma matemática. Para darle un sentido lúdico a la actividad y motivar la participación, el primer equipo en tener la respuesta completa, debería mandar un representante al frente para sentarse sobre un globo y romperlo. Parecería banal esta actividad, pero para ellos fue el detalle que marcó la diferencia; el mero hecho de desear romper el globo los hizo apoyarse en equipo, estar todos atentos en dar con las respuestas y luego correr rápidamente y reventar el globo. Risas, 
mentes trabajando, colaboración, son aspectos valiosos para todo profesor interesado no sólo en el aprendizaje de la matemática.

Posteriormente, se procedió a revisar las diferentes maneras en que se habían logrado las respuestas.

Continuando con el plan de clase, otro día se realizó una actividad parecida a la anterior, solo que en esta ocasión se escribían en el pizarrón dos respuestas y los alumnos debían elegir la correcta. Se organizaron en equipos. Nuevamente, para motivar la participación de los alumnos, se recurrió a un juego que consistía en golpear con una mano didáctica la respuesta correcta escrita en el pizarrón (solo pasaría un representante del equipo), recordándoles la importancia de los dos aspectos, la respuesta correcta por un lado y la expresión formal del problema mediante el uso de ecuaciones de primer grado, por el otro.

La participación de los alumnos fue gratificante para nosotros, ya que con gran entusiasmo, risas $y$ prisas trataban de terminar lo más pronto posible para tomar la mano didáctica y golpear el pizarrón en donde estaba la respuesta correcta.

Una vez que la mayor parte de los equipos había terminado el ejercicio, se procedió a revisar las diferentes soluciones, y se encontró que varias de ellas eran correctas y que se habían hecho en algunos casos razonamientos distintos, mientras que en otros aparentemente exitosos se detectaron errores por parte de los mismos alumnos. A partir de los deficientes resultados obtenidos en el examen diagnóstico, se aprovechó para reforzar los aspectos correspondientes de la aritmética y del álgebra que fueron usados.

Le agradaron ejercicios referidos a la cantidad de novios y novias, hamburguesas que consumen, calorías y energías utilizadas, entre tantos más.

Otro día acudimos al centro de cómputo para navegar en Internet y mostrar a los alumnos que existen programas gratuitos en los que se pueden encontrar actividades entretenidas que facilitan el aprendizaje 
de las matemáticas. También les dimos el software de Álgebra donde divertidamente se adentraban en el mundo de las matemáticas y en específico en álgebra. Hacían ejercicios, creaban problemas cotidianos y los expresaban algebraicamente, los socializaban y resolvían de diferentes maneras.

Reiteradamente se aprovechó la oportunidad para señalar la importancia que tienen la imaginación y la creatividad en la solución de los problemas, haciendo notar también que las ecuaciones no son más que otras maneras de expresión y que nosotros podemos crear y recrear nuestras fórmulas para conocer mejor el mundo que nos rodea.

La evaluación, el postest.

Se aplicó un examen del tema de ecuaciones de primer grado a los alumnos con los que trabajábamos, el grupo $D$, al cual se le denomina grupo piloto experimental o control. También se aplicó el mismo examen a los alumnos del segundo $E$, que se designó grupo testigo para que sirviera como punto de referencia o comparación. El maestro del grupo D también imparte clases en el E, y fue quien nos asignó a los dos, el primero como control y el segundo como testigo.

Considerando el marco del constructivismo en el diseño del examen, se le dio más peso al aspecto del planteamiento y solución de problemas para tratar de determinar si habíamos logrado incidir de alguna manera en los alumnos con nuestro trabajo. El examen constó de cuatro reactivos, tres de los cuales fueron ejercicios de planteamiento y solución de problemas y uno de contenido aritmético.

Las calificaciones del grupo testigo, -solo un alumno consiguió calificación aprobatoria-, se encuentran por debajo de las calificaciones del grupo piloto (experimental), en el cual lograron aprobar cuatro alumnos.

Del grupo piloto, 39 alumnos obtuvieron calificación reprobatoria y 4, aprobatoria, También se tiene que la calificación mínima es de 0 y fue obtenida por seis alumnos. La calificación máxima es de 9.6 y fue obtenida solamente por un alumno. Con estos logros, resulta claro que 
el promedio de calificación para el grupo es reprobatorio: 3.34. Esta baja calificación promedio del grupo piloto se debe en parte a que el reactivo cuatro, que es esencialmente aritmético, no fue contestado de manera correcta por ningún alumno, cuando se esperaba que fuese contestado correctamente por la mayoría de ellos.

Lo anterior confirma la imagen obtenida con base en el examen diagnóstico del bajo nivel académico de los alumnos, quienes arrastran deficiencias de los niveles escolares previos.

En cuanto al grupo testigo, éste obtuvo una calificación promedio de 2.12 , la cual fue inferior a la lograda por el grupo piloto. Asimismo, 26 alumnos del grupo piloto lograron resolver correctamente alguno de los ejercicios que implicaban planteamiento para su solución. En cambio, del grupo testigo solamente 5 alumnos lograron hacerlo. Pero esto no debe conducirnos a concluir precipitadamente que el impacto logrado por nuestro trabajo es notablemente positivo. Si bien es cierto que estamos convencidos de que con nuestro quehacer logramos incidir tanto en la actitud como en el conocimiento de los jóvenes del grupo piloto, también es cierto que en tan sólo dos semanas de actividades no existe evidencia suficiente para cuantificar de manera clara la magnitud del impacto logrado con nuestra dinámica.

\section{DiSCUSIÓN DE RESULTADOS Y CONCLUSIONES}

Las nobles obras inician con francas aspiraciones, y esa fue la razón de nuestro proceder: crear, modificar, experimentar y mejorar, pero como se observó, nuestros resultados no fueron tan alentadores como esperábamos.

Ciertamente, logramos disminuir un poco el índice de reprobación, en tanto que nuestro grupo reprobó menos que el grupo testigo, a quienes no se les aplicó la propuesta, aunque revisaron los mismos contenidos con el maestro titular de la materia. Sin embargo, la disminución no fue tan significativa, porque no esperábamos que los estudiantes del grupo piloto reprobaran.

Las razones de esos corolarios 
son hasta cierto punto simples. Como dice la famosa frase, "Ios cambios no se dan de la noche a la mañana". Circunspectos en esa realidad, podemos aducir que sí logramos mucho y que lo vivido y experimentado tiene una valía incalculable. No es lo mismo planear, imaginar y elaborar una propuesta detrás del escritorio, que llevarla a las aulas con alumnos reales (con intereses y motivaciones peculiares) que fácilmente se distraen, que no participan si no hay puntos extra en sus calificaciones... y, además, con una propuesta didáctica que poco ha sido empleada sistemáticamente e inspeccionada y regulada durante toda su ejecución.

Fueron muy pocas las sesiones. Para transbordar el constructivismo a las aulas es necesario suficiente tiempo, incluso quizás todo el curso (y eso quizás no sea garantía de éxito). Por años los alumnos en general han aprendido a mecanizar la matemática, a ser motivados con estímulos externos, pero también a ser regañados, callados, apresurados, a hacer los ejercicios siguiendo el procedimiento elegido por enseñante. Comprobamos que han aprendido la matemática mecánica- mente: la repetición de lo que se debe aprender y el ejercicio. Tanto repite y ejercita el alumno, que consigue realizar las actividades con un alto grado de automatismo, pero cuando se trata de integrar los saberes a una situación diferente, tal vez real, muy pocos pueden hacerlo efectivamente. La automatización se alcanza, pero no la construcción propia. Eso fue muy claro, la construcción les parecía un reto que casi nadie quería enfrentar, mientras que la mecanización era sencilla, y "fácilmente" podían encontrar el resultado.

El constreñido tiempo obstruyó los enormes deseos: la construcción, la facilitación y andamiaje, la recuperación de conocimientos previos, uso e incremento de la zona de desarrollo próximo e implementación de las tecnologías de la información y la comunicación.

Pese a todo, se confirma completamente la segunda parte del supuesto hipotético: las matemáticas pueden aprenderse de manera divertida. Con entusiasmo, los alumnos esperaban el comienzo de la clase de matemáticas, sus ojos cu- 
riosos los delataban, miraban el material didáctico preparado para el tema del día, preguntaban "¿qué vamos hacer hoy?", y se involucraban con gran motivación en todas las actividades previamente planeadas. Lo anterior nos lleva a inferir que la construcción significativa del contenido de la enseñanza es tan importante como la significación que le otorgan los alumnos a la propia situación didáctica, la cual pueden percibirla como motivante, retadora 0 , por el contrario, inabordable y abrumadora, desprovista de interés o inalcanzable para sus posibilidades (Coll, Miras, Onrubia, Solé y Zabala, 2007). Por eso cuidamos en todo momento que las actividades fueran eminentemente didácticas, supusieran retos no abrumadores sino motivantes e inquietantes, adaptados al nivel cognitivo de los estudiantes. Sin descuidar el contenido, la dinámica de grupo creada condujo a que en equipo reflexionaran los procesos y respuestas, y las emitieran con prontitud a través del juego competitivo. Quizás esto no hubiera funcionado con adultos e incluso con jóvenes, pero para esta etapa de desarrollo, la adolescencia, tal dinámica fue fructuosa, ya que en menos tiempo emitían sus respuestas consensuadas en equipo, las cuales eran contrastadas con las de los demás. Todos participaban, en especial cuando había juegos. Les parecía asombroso que se pudiera jugar y al mismo tiempo se aprendiera álgebra.

Otros mecanismos idóneos fueron los medios tecnológicos, presentaciones en power point, software y CDs, y las páginas en internet; todo de gran valía para reforzar el aprendizaje del álgebra de manera entretenida.

En efecto, fueron pocos días de clases, pero nos dejaron exhaustos: ponernos de acuerdo, planear cómo dar la clase sin salirse de las bases del constructivismo, adaptar los ejercicios a las características del alumnado, construir el material didáctico, entre tantas acciones más. Para dar menos de una hora de clase, nos llevamos tres horas en prepararla; pero veíamos cambios que nos alentaban, el interés mostrado de los alumnos, su implicación en las tareas, sus risas y emociones... por eso cuando pensamos en informar nuestro resulta- 
dos no quisimos recurrir al tradicional reporte de investigación, sino a la experiencia compartida.

\section{REFERENCIAS}

Ausubel, D. (1976) Psicología educativa. Un punto de vista cognitivo. México:

Trillas.

Coll, C. Miras, Onrubia, Solé y Zabala (2007) El constructivismo en el aula. Barcelona: Graó.

Dewey, J. (1958). Experiencia y educación. Buenos Aires: Losada

Díaz Barriga F. y Hernández (2002)

Estrategias docentes para un aprendizaje significativo. Una interpretación constructivista. México: Mc Graw Hill.

Freinet, C. (1964) Las enfermedades de la escuela. Barcelona: Laia, 1974 (traducción)

Good y Brophy (1994) "Empathic: An unappreciated way of being", en Hernández, Juaréz, Landgrave y Mendoza (2012) Enseñanza constructivista del álgebra. Narrativa de cuatro voces. México: Arana.

Hernández G. (2011) Práctica docente más allá de cuatro paredes, pizarrón y mesabancos. México: Arana.

Hernández G, Juárez, Landgrave y Mendoza (2012) Enseñanza constructivista del álgebra. Narrativa de cuatro voces. México: Arana

La Gaceta ( $1^{\circ}$ de julio de 2002) Xalapa, Veracruz.

Perking, D. (1995) La escuela inteligente: el adiestramiento de la memoria a la educación de la mente:

Barcelona: Gedisa.

Rodrigo M. y Cubero (1998)

Constructivismo y enseñanza: reconstruyendo las relaciones en ciencia. Madrid: Aprendizaje visor.

Roger. C. (1991) Libertad y creatividad en la educación en la década de los 80 s. Barcelona: Paidós.

Secretaría de Educación Pública (2007). Programa Sectorial de Educación 20072012. Consultado en www.sep.gob.mx el 21 de enero de 2009. 